\title{
28 And: Simultaneous Strömgren Photometry
}

\author{
E. Rodríguez ${ }^{1}$, A. Rolland ${ }^{1}$, P. López de Coca $^{1}$, R. Garrido ${ }^{1}$ \\ \& E.E. Mendoza ${ }^{2}$ \\ ${ }^{1}$ Instituto de Astrofísica de Andalucia, Apartado 3004, 18080 Granada, Spain \\ ${ }^{2}$ Inst. de Astron., UNAM, Apartado Postal 70-264, CP-4510, México D.F., México
}

\begin{abstract}
We have carried out simultaneous uvby photometry of the low amplitude $\delta$ Sct star 28 And. Analysis of the data, using the Fourier Transform method, establishes 28 And as a monoperiodic pulsator. Using the classical O-C method, it is found that the pulsation of this star can be well described by means of a linear ephemeris with a period of $\mathrm{P}=0 .{ }^{d} 069304118$ over the last twenty-four years. Amplitude variations are also shown to be present from season to season. The physical parameters of this star are determined and the nature of radial or nonradial pulsation is discussed on the basis on the derived phase shifts and amplitude ratios between Strömgren colours. The results indicate that 28 And pulsates in a nonradial mode with $\ell=2$.
\end{abstract}

\section{$\rho$ Pup: A Monoperiodic Radially Pulsating $\delta$ Sct Star}

\author{
Eloy Rodríguez, Angel Rolland \& Pilar López de Coca \\ Instituto de Astrofísica de Andalucía, \\ Apartado 3004, 18080-Granada, Spain.
}

\begin{abstract}
$\rho$ Pup is a $\delta$ Scuti type pulsator with relatively low amplitude $\left(\Delta V \sim 0 .^{m} 09\right)$. Analysis of the data from different sources, using the Fourier Transform method, establishes $\rho$ Pup as a monoperiodic pulsator. Using the classical O-C method, it is found that the pulsation of this star can be well described by means of a linear ephemeris with a period of $\mathrm{P}=0 .{ }^{d} 140881372$ over more than eighty years. The nature of radial or nonradial pulsation is discussed on the basis on the derived phase shifts and amplitude ratios between different colours. The results indicate that $\rho$ Pup is a radial pulsator.
\end{abstract}

